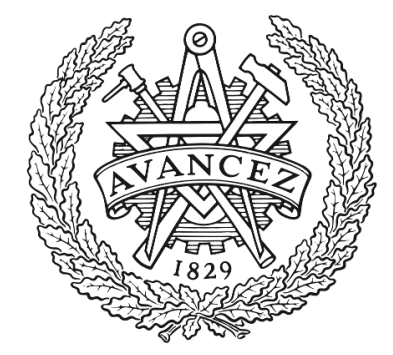

\title{
CHALMERS
}

UNIVERSITY OF TECHNOLOGY

\section{Slot Antenna Array Unit Cell Directly Fed by Inverted Microstrip Gap Waveguide}

Downloaded from: https://research.chalmers.se, 2023-04-26 12:44 UTC

Citation for the original published paper (version of record):

Liu, J., Vosoogh, A., Uz Zaman, A. et al (2017). Slot Antenna Array Unit Cell Directly Fed by Inverted Microstrip Gap Waveguide. 2017 International Symposium on Antennas and Propagation (ISAP). http://dx.doi.org/10.1109/ISANP.2017.8228975

N.B. When citing this work, cite the original published paper. 


\title{
Slot Antenna Array Unit Cell Directly Fed by Inverted Microstrip Gap Waveguide
}

\author{
Jinlin Liu, Abbas Vosoogh, Ashraf Uz Zaman, and Jian Yang \\ Department of Electrical Engineering, Chalmers University of Technology, Gothenburg, Sweden \\ Jinlin.Liu@chalmers.se
}

\begin{abstract}
Inverted microstrip gap waveguide is mechanically flexible millimeter wave transmission line. This paper describes a single-layer slot antenna array unit cell at $60-\mathrm{GHz}$ designed by using this inverted microstrip gap waveguide technology. This antenna unit mainly consists of a $2 \times 2$ slot layer and corporatefeed networks. The unit-cell shows more than $14.6 \%$ bandwidth over 57-66 GHz band with 10-dB return loss. Infinite array environment has been utilized later to determine overall efficiency for bigger $16 \times 16$ antenna array which is around $70 \%$ with a directivity value of $33.5 \mathrm{dBi}$ between 57 - $66 \mathrm{GHz}$.
\end{abstract}

Index Terms-Artificial Magnetic Conductor (AMC), gap waveguide, high efficiency, millimeter wave, slot array antenna.

\section{INTRODUCTION}

Currently, the $60-\mathrm{GHz}$ frequency band has gained increased attention in the research community due to its potential benefits for many upcoming applications, such as video streaming, high-speed Internet, high-definition multimedia interface, wireless gigabit Ethernet, etc. One of the key elements for such wireless applications is the antenna, which should be able to provide high gain, high radiation efficiency, and wide bandwidth characteristics. Consequently, planar array antenna is a suitable candidate. Microstrip antenna arrays and waveguide slot arrays are the two main planar antenna technologies, which have been used extensively over a wide range of frequencies. The microstrip feed networks suffer from high ohmic and dielectric losses at high frequency. Spurious radiations and leakage in the form of surface waves are also major concerns in microstrip antennas. On the other hand, waveguide slot arrays have lower loss and high efficiency. However, wide band waveguide slot arrays require corporate feed networks that are mechanically very complex. At high frequencies, such networks require accurate (and thereby expensive) manufacturing so as to achieve good electrical contacts between the slotted metal plate and the bottom feed structure.

Based on the above discussion, existing corporate-feed network technologies have limitations with respect to bandwidth and mechanical simplicity, giving an opportunity for the proposed gap waveguide technology [1]. The gap waveguide technology utilizes the basic cutoff of a perfect electrical conductor, perfect magnetic conductor (PEC-PMC) parallel-plate waveguide configuration to control desired electromagnetic propagation between the two parallel plates [1]. In the realized gap waveguide, the PEC-PMC cutoff can be achieved by a periodic structure, such as metal pins or mushrooms surfaces. However, the textured surface must also incorporate guiding structures in the form of ridges, grooves or strips. These guiding structures thereby define three different gap waveguides or transmission lines, referred to as ridge, groove and inverted microstrip gap waveguides [2]. The present paper deals with inverted microstrip gap waveguide. Consequently, gap waveguide technology offers mechanically flexible guiding structures where good electrical contact between the metallic blocks is not an issue at all. Until now there have already been lots of applications of gap waveguide technology for MMIC packaging [3] antennas [4]-[7] and filters [8]. We should also mention here that all types of antennas in [4]-[7] are multilayered. In the present paper, a unit cell for slot antenna array directly fed by inverted microstrip gap waveguide will be introduced.

\section{ANTENNA CONFIGURATION}

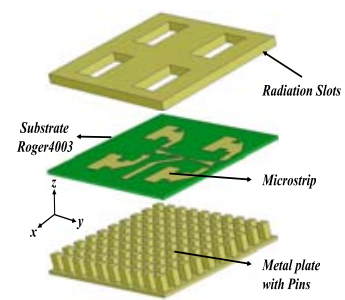

Fig. 1. Detailed 3-D view of single-layer slot array unit cell.

The $2 \times 2$ slot array unit cell is illustrated separately in 3D view in Fig. 1. The structure briefly consists of three layers. The top layer contains four radiating slots. These four radiating slots is directly fed by the distribution networks, which have been designed by using the inverted microstrip gap waveguide. Below the PCB, there is a bottom metal layer with a uniform grid of pins. The radiating slots are uniformly spaced in both $x$ and $y$ directions. Their distances are kept less than one wavelength in order to minimize the grating lobe level and at the same time the element spacing are optimized to get the highest possible directivity of a large $16 \times 16$ slots array. The size of the radiating slots is $2.5 \times 1.37 \mathrm{~mm}^{2}$ and the distances between every two slots in $x$ and $y$ directions are $4.5 \mathrm{~mm}$. The designed unit cell has the dimensions of $9 \times 9 \mathrm{~mm}^{2}$ in both 


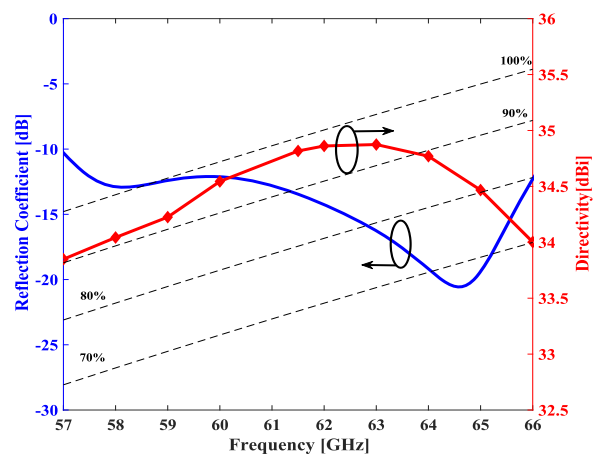

Fig. 2. Simulated directivity and reflection coefficient of $2 \times 2$ sub-array.

E- and H- planes respectively. The pins in the metal plate have the dimensions of $0.5 \times 0.5 \times 0.9 \mathrm{~mm}^{3}$. The period of pins has been kept $0.9 \mathrm{~mm}$ in both E- and H-planes respectively. The air gap between substrate and metallic lid is chosen equal to $0.2 \mathrm{~mm}$. Roger 4003 with the thickness of $0.254 \mathrm{~mm}$ is utilized as the substrate for inverted microstrip feed line.

\section{SIMULATED RESULTS}

In numerical simulation, the $2 \times 2$ element slot array is excited by an optimized waveguide port [9] at the inverted microstrip gap waveguide. The simulated reflection coefficient of the designed unit cell is shown in Fig. 2. We observe that $S_{11}$ is below $-10 \mathrm{~dB}$ over the frequency range $57-66 \mathrm{GHz}$.

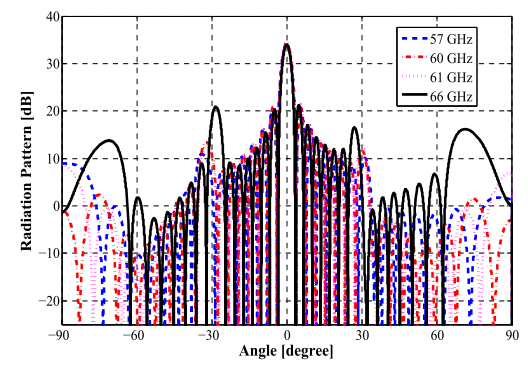

Fig. 3. Simulated radiation patterns in E-plane for $16 \times 16$ slot array.

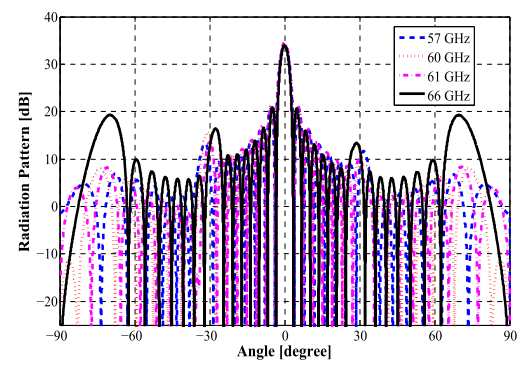

Fig. 4. Simulated radiation patterns in H-plane for $16 \times 16$ slot array.
The antenna unit cell has been simulated in an infinite array condition with periodic boundary condition. The simulated radiation patterns for the $16 \times 16$ slot array in $\mathrm{E}$ and $\mathrm{H}$-plane are shown in Fig. 3 and Fig. 4, respectively. We have observed that the grating lobe levels (GL) in E- and H-planes becomes high at $66 \mathrm{GHz}$. The main reason for this problem is that the wavelength $\lambda[\mathrm{f}=66 \mathrm{GHz}]=4.55 \mathrm{~mm}$ is almost identical to the distance between two slots in $x$ and $y$ directions (4.5 $\mathrm{mm}$ ). Also, the space for feed networks is so limited between the air gap that the mutual coupling effect between the lines of the feed networks is ineluctable. Thereby, the radiation pattern of single-layer antenna is a little bit unsymmetrical. The simulated aperture efficiency of the $16 \times 16$ slot array is higher than $70 \%$.

\section{CONCLUSION}

In this work we have numerically designed the radiating elements of a single-layer planar slot array unit cell at 60 $\mathrm{GHz}$. The inverted microstrip gap waveguide is applied for the feeding. Full-wave simulated results show promising results over the bandwidth over 57.7 ? $65.7 \mathrm{GHz}$. The proposed antenna unit cell is suitable for $60-\mathrm{GHz}$ applications.

\section{REFERENCES}

[1] P.-S. Kildal, E. Alfonso, A. Valero-Nogueira, and E. Rajo-Iglesias, "Local metamaterial-based waveguides in gaps between parallel metal plates, "IEEE Antennas Wireless Propag. Lett., vol. 8, pp. 84-87, 2009.

[2] P.-S. Kildal, "Three metamaterial-based gap waveguides between parallel metal plates for $\mathrm{mm} / \mathrm{submm}$ waves, " in 3rd European Conference on Antennas and Propagation, EuCAP 2009, pp. 28-32.

[3] A. U. Zaman, V. Vassilev, P. S. Kildal and H. Zirath, "Millimeter Wave E-Plane Transition From Waveguide to Microstrip Line With Large Substrate Size Related to MMIC Integration, "IEEE Microwave and Wireless Components Letters, vol. 26, no. 7, pp. 481-483, July 2016.

[4] A. Vosoogh and P.-S. Kildal, "Corporate-Fed Planar $60 \mathrm{GHz}$ Slot Array Made of Three Unconnected Metal Layers Using AMC pin surface for the Gap Waveguide, "IEEE Antennas and Wireless Propagation Letters, December, 2015

[5] A. Jimnez Sez, A. Valero-Nogueira, J. I. Herranz and B. Bernardo, "Single-Layer Cavity-Backed Slot Array Fed by Groove Gap Waveguide, "?IEEE Antennas and Wireless Propagation Letters, vol. 15, pp. 14021405, 2016.

[6] D. Zarifi, A. Farahbaksh, A. Uz Zaman and P.-S. Kildal, "Design and fabrication of a wideband high-gain $60-\mathrm{GHz}$ corrugated slot antenna array with ridge gap waveguide distribution layer, "IEEE Transactions on Antennas and Propagation, vol. 65, no. 4, pp. 2117-2122, April 2017.

[7] J.L. Liu A. Vosoogh A. U. Zaman and J. Yang, "Design and Fabrication of a High Gain 60-GHz Cavity-backed Slot Antenna Array fed by Inverted Microstrip Gap Waveguide, "IEEE Trans. Antennas Propag., vol. 65, issue: 4, April, 2017.

[8] A. Vosoogh, A. A. Brazalez and P.-S. Kildal, "A V-Band inverted microstrip gap waveguide end-coupled bandpass filter, "IEEE Microwave and Wireless Components Letters, vol. 26, no. 4, April, 2016.

[9] J. L. Liu, A. Uz Zaman and P.-S. Kildal, "Optimizing numerical port for inverted microstrip gap waveguide in full-wave simulators, "Antennas and Propagation (EUCAP), Proceedings of the 10th European Conference on, 10-15 April 2016. 